\title{
INFECTION AND THROMBOTIC COMPLICATIONS IN PERIPHERALLY INSERTED CENTRAL CATHETERS (PICCS) IN CANCER PATIENTS AT HEALTHCARE GLOBAL HOSPITAL, BANGALORE
}

\author{
Sapkota $S^{1^{*}}$, Priyadarshini $M^{2}$, Sannur $R^{3}$, Naik $R^{4}$
}

\author{
Affiliation \\ 1. Assistant Professor, Medical Oncology, Birat Medical College, \\ Biratnagar, Nepal \\ 2. Consultant, Obstetric and Gynaecology/Gyanae-Oncology, Birat \\ Medical College, Biratnagar, Nepal \\ 3. Clinical specialist, Nursing Department, HCG Enterprises Ltd, \\ Bangalore, India. \\ 4. HOD, Medical Oncology \& Hematology Department, HCG \\ Enterprises Ltd, Bangalore, India.
}

\section{ARTICLE INFO}

Received : 26 January, 2020
Accepted : 23 March, 2020
Published : 30 June, 2020

Published : 30 June, 2020

(c) Authors retain copyright and grant the journal right of first publication with the work simultaneously licensed under Creative Commons Attribution License CC - BY 4.0 that allows others to share the work with an acknowledgment of the work's authorship and initial publication in this journal.

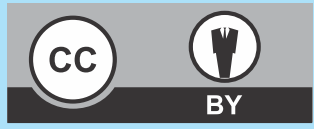

\section{ORA 162}

DOI: https://doi.org/10.3126/bjhs.v5i1.29631

\section{* Corresponding Author \\ Dr. Sulav Sapkota \\ MD (Medical Oncology)}

Fellowship (Hemato-Oncology \& Bone Marrow Transplant)

Assistant Professor, Birat Medical College Teaching Hospital, Nepal Email ID: dr.sulavsapkota@yahoo.com

ORCID ID: https://orcid.org/0000-0003-4894-6455

\section{Citation}

Sapkota S, Priyadarshini M, Sannur R, Naik R. Infection And Thrombotic Complications In Peripherally Inserted Central Catheters (PICCS) In Cancer Patients At Healthcare Global Hospital, Bangalore. BJHS 2020;5(1)11: 946-949.

\section{ABSTRACT}

\section{Introduction}

Peripherally inserted central catheter (PICC) is a form of vascular access device (VAD) that can be used for a prolonged period of time in cancer patients for diverse indications. Despite the many advantages, catheter related complications are common.

\section{Objectives}

The objective of this study was to evaluate infection and thrombotic complications of PICCS in cancer patients.

\section{Methodology}

We conducted a prospective longitudinal study, over a duration of thirty months from June 2015 to December 2017 in the Department of Haematology and Medical Oncology at our tertiary care, cancer hospital to assess, analyze and compare the infection and thrombotic complications in cancer patients with a PICC line. All patients with cancer who met the inclusion criteria were enrolled and were followed up until catheter removal. The basic information regarding PICCs insertion, weekly care and removal was maintained. The data were analyzed and compared to identify catheter related complications

\section{Result}

Four hundred and sixty-one PICCs were monitored for a total of 57,438 catheter-days (mean of 124 days i.e. 4.15 months, range : 1 to 434 days). Out of these 461 PICCs, 29 (6.29\%) had both infection and thrombotic complications, as a result of which 22 were removed in 57,438 catheter days. (ie catheter removal rate was 0.38 per 1000 PICCdays). Infection complications were found more with hematological malignancies while thrombotic complication were predominant in solid tumours .

\section{Conclusion}

Infection complications were more common in hematological cancer patients than solid cancers where thrombotic complications were more common.

\section{KEYWORDS}

Infection; peripherally inserted central catheter (PICC); thrombotic 


\section{INTRODUCTION}

A peripherally inserted central catheter (PICC) is a form of vascular access device (VAD) which was first described in 1975. It is an alternative to centrally inserted central catheter, which was related to a higher rate of complications. ${ }^{1}$ PICCs provide reliable and relatively safe intravenous access in varied indications for delivery of any infusate like chemotherapy and other intravenous fluids irrespective of $\mathrm{pH}$, osmolarity, or other chemical properties of the solution or medication. ${ }^{2}$ Many intravenous medications cause direct chemical irritation and vascular endothelial damage, but these drugs when infused through a PICCs are immediately diluted at the central line tip by the high blood flow around the catheter which helps protect the vessel wall. ${ }^{3}$ PICCs are made of either silicone or polyurethane, with the former being associated with lesser risk of thrombosis. ${ }^{4}$

Two major complications that occur with central venous access including PICCs are infection and thrombosis. The types of infection related to PICCs are either superficial, involving the venous puncture site, also known as cellulitis or those involving the blood stream i.e. catheter related bloodstream infection (CRBSI). Cellulitis is generally a mild to moderate form of infection, which can be treated with regular local dressing, local or oral antibiotics, on the other hand CRBSI is a severe form warranting hospitalisation and intravenous antibiotics. During the diagnosis of CRBSI, the blood culture is found to be positive but at the same time the sample from PICCs may not show any growth. It may lower the quality of life, adds to the financial burden and even contribute to the mortality. ${ }^{5}$ Still, as compared to the traditional CVCs, PICCs show lower risk of blood stream infection. $^{6,7,8}$

On review of literature, infection rate related to PICCs shows varied results. Alenjo et al. (2011) observed higher PICC days (4.79 per 1000 PICC days) in ICU patients than the non- ICU group (2.78 per 1000 PICC days). ${ }^{9}$ Similar result was observed by Chopra et al. (2014) where they identified ICU as a risk factor for infectious complications with a PICC-BSI rate of 2.16 per 1000 PICC-days. ${ }^{10}$ Gao, Y. et al (2015) in their study specified that fixing method, indwelling season, catheter care and tip position were significantly associated with the incidence of PICC related bloodstream infection. ${ }^{11}$ In a recent study conducted by Delphine grau et al (2017), the overall complication rate was $30.2 \%$, which was higher in the hospitalization setting than in the outpatient setting. ${ }^{12}$ Herc E et al (2017) in an another study have tried to develop a model to predict central line associated bloodstream infection among patients with peripherally inserted central catheters : The MPC score. ${ }^{13}$

Catheter related venous thrombosis is because of direct release of thrombogenic factors, by the tumour cells, tumour induced decreased antithrombotic natural factors, and procoagulant activity of many chemotherapy drugs. The material and calibre of the lumen of the catheter has also been noticed to have an effect on the thrombosis rate. ${ }^{14}$

Hence we wanted to conduct a study in our department to evaluate infection and thrombotic complications of PICCs in our cancer patients for feasibility of incorporating into inhouse protocol.

\section{METHODOLOGY}

We conducted a prospective longitudinal study from June 2015 to December 2017 in our tertiary comprehensive cancer hospital. Ethical clearance was obtained from the department. Informed written consent was taken from all of the cancer patients enrolled in the study. The inpatients in whom the venous access was difficult were referred to our PICC centre for PICC insertion. Under ultrasound guidance, $4 \mathrm{Fr}$ single lumen triple-valved PICC was inserted with strict aseptic care. The PICC was inserted in any of the major vein of the upper extremities, more frequently in basilic vein and secured by stat-lock adhesive dressing. Weekly follow-up for these patients were arranged with the PICC team in a ward or minor operation theatre for dressing.

CRBSI was defined as patients with PICCs presenting with fever with chills, unexplained hypotension and no other localising signs along with positive blood culture.

Catheter-related venous thrombosis was defined by detection of thrombus by ultrasonography at intraluminal, extraluminal or at the tip of catheter site.

Patients with histologically confirmed malignancy , having good performance status and who agree to give consent were included in the study while patients with coagulopathy, superior venacaval syndrome were excluded.

Pro forma was maintained that included the demographic profile of the patients, such as name, age, sex, and all the parameters related to the PICCs such as date of insertion and removal of the PICC, diagnosis of patients for which PICC inserted, patient status, type of vein accessed, complication (if any), PICC dwell days etc. Data analyses were performed using SPSS software. Demographic characteristics were summarized by descriptive statistics. The rates of complications were expressed by percentage and per 1,000 catheter days. For the analysis, each PICC placement was counted as a new event. In case of lost to follow up, last visit was taken as the date of removal of PICC lines.

\section{RESULTS}

A total of 461 PICCs were inserted successfully during the study period, of which four PICCs were lost to follow up as depicted in Table 1 .The study included 225(49\%) men and $236(51 \%)$ women with a mean age of 47.8 years(range :7-84 years). Of the study population , $61 \%$ had solid malignancies and $39 \%$ had hematological malignancies. Among the solid tumours, the most common was GIT malignancy while lymphoma was the most common indication in the hematological group. Most frequently used vein was basilic for PICC placement .

The 461 PICCs were in place for a total of catheter 57,438 days (mean of 124 days, i.e. 4.15 months and range of 1 to 434 days). The most common indication for PICC was chemotherapy. Of these, 29 (6.29\%) PICCs had combined infection and thrombotic complications of which 22 PICCs were removed at the rate of $0.38 / 1000$ PICC-days due to complications. It was seen that infection complications with PICCs were more prevalent in the patients with hematological 
malignancies while thrombotic complications were seen more frequently with solid tumours.

\begin{tabular}{|c|c|c|}
\hline Characteristics & No. of patients & Percentage \\
\hline \multicolumn{3}{|l|}{ Age (years) } \\
\hline $07-14$ & 04 & 0.9 \\
\hline $15-24$ & 27 & 5.9 \\
\hline $25-34$ & 73 & 15.8 \\
\hline $35-44$ & 65 & 14.8 \\
\hline $45-54$ & 99 & 21.4 \\
\hline $55-64$ & 132 & 28.6 \\
\hline $65-74$ & 50 & 10.8 \\
\hline $75-84$ & 8 & 1.8 \\
\hline Mean Age & 47.8 & - \\
\hline Male & 225 & 49 \\
\hline Female & 236 & 51 \\
\hline \multicolumn{3}{|l|}{ Underlying Cancers } \\
\hline Solid Tumors & 282 & 61 \\
\hline Hematological cancer & 179 & 39 \\
\hline \multicolumn{3}{|l|}{ Complications } \\
\hline CRBSI & 8 & 1.6 \\
\hline Cellulitis & 8 & 1.8 \\
\hline Combined CRBSI and Cellulitis & 16 & 3.4 \\
\hline Thrombosis & 13 & 2.82 \\
\hline $\begin{array}{l}\text { Total PICCs removed due to } \\
\text { infection and thrombosis. }\end{array}$ & 22 & 4.77 \\
\hline Lost to follow up & 4 & 0.87 \\
\hline
\end{tabular}

\section{Characteristics of Infection Complications:}

Infection complications, including both superficial infection (Cellulitis) and CRBSI (catheter related blood stream infections) were the most common complication in our study. Total 16 out of 461 patients (3.4\%) had infections with the incidence rate of 0.27 per thousand catheter days. Infection complications included 8 CRBSI and 8 Cellulitis. The majority of the CRBSI patients presented to the hospital with high grade fever with chills and rigour and to establish the diagnosis blood culture was done in these patients. Minimal two culture bottles, one from PICC and another from peripheral vein sample were sent to the microbiology department. During the study, $8(1.6 \%)$ patients had positive blood cultures out of whom $2(0.43 \%)$ cases showed growth at the PICC tip and 2 patients showed growth both at the PICC and peripheral vein sample. The most common organism which grew on blood culture were coagulase negative staphylococcus and pseudomonas aeruginosa which were sensitive to commonly used antibiotics. Another common presentation was cellulitis where patients presented with local infection of the skin with features like redness, warmth, rashes etc. It was mainly diagnosed clinically without culture. Antibiotics were used as per the antibiogram for CRBSI while oral antibiotics were used for the treatment of cellulitis. It was possible to salvage 4 infected patients while 12 cases could not be rescued with antibiotics, hence the PICC removed.Those salvageable mainly presented with cellulitis. Fever usually subsided within forty-eight hours of removal of PICC line in CRBSI.

\section{Characteristics of thrombotic complications:}

Thrombotic complications were next most common complications to infection, with the rate of incidence of 0.22 per thousand catheter days. Total 13 out of 461 patients
$(2.82 \%)$ developed catheter related thrombosis. The main feature of the presentation was pain and swelling of the hand or arm. A venous doppler ultrasound was performed for the confirmation of diagnosis and the patients were started on low molecular weight heparin till thrombosis resolved and later PICC line was removed. Total 3 thrombotic cases were salvageable with the use of anticoagulant while 12 PICCs were removed. Clinical features usually subsided within a week after the removal of PICC lines. During the study, one patient needed surgical removal of the thrombus from his arm by the orthopaedic surgeon.

\section{DISCUSSION}

PICCs are now being used more commonly in cancer settings due to ease of insertion and low procedure related complications. However the infection and thrombotic complications related to PICCs in cancer patients have not been studied in detail in the Indian population, therefore we prospectively evaluated these complications in our study. In our study, we compare the infection and thrombotic complications as depicted in Table 2. It was observed that infection complications were common in the younger age group with age $<45$ years, while thrombotic events were more in the elderly patients. 10 out of 16 patients (62.5\%) showed infective complications more commonly seen in those with hematological cancers, this could be due to the aggressive nature of cancers, use of multiple chemotherapy drugs, prolonged steroid use and multiple blood and blood products infusions. Nine out of 13 patients (69.2\%) had thrombotic events, mainly seen in patients with solid cancers, which could be explained by the thrombogenic nature of cancers and age related factors. The mean number of attempts for skin puncture during PICC insertion were similar ( 1 attempt) in both infected and thrombotic groups with similar mean duration of time for PICCs insertion. The mean depth of PICCs inserted into the arms (approx $1 \mathrm{~cm}$ ) and the length of PICCs (approx. $38 \mathrm{~cm}$ ) were similar in both cases. There were no deaths due to infection and thrombosis of PICCs during the study period as PICCs were removed immediately after the complications.

Table 2: Infection and thrombotic complication analysis.
\begin{tabular}{|l|c|c|}
\hline \multicolumn{1}{|c|}{ Characteristics } & $\begin{array}{c}\text { Infection } \\
\text { complications }\end{array}$ & $\begin{array}{c}\text { Thrombotic } \\
\text { complications }\end{array}$ \\
\hline Incidence rate per thousands catheter days & 0.27 & 0.22 \\
\hline Incidence rate in percentage & 3.47 & 2.82 \\
\hline Male patients & 9 & 6 \\
\hline Female patients & 7 & 7 \\
\hline Solid Tumors cases & 6 & 9 \\
\hline Hematological cases & 10 & 4 \\
\hline Salvageable cases & 4 & 3 \\
\hline Non salvageable cases & 12 & 10 \\
\hline Age <45yrs & 9 & 4 \\
\hline Age>45 yrs & 7 & 9 \\
\hline Mean number of attempts & 1.07 & 1.18 \\
\hline Mean duration of PICC insertion(minutes) & 25 & 27.25 \\
\hline Most common vein(LT Basilic) & 10 & 11 \\
\hline Mean Depth of PICC (cm) & 0.9 & 1.0 \\
\hline Mean length of PICC (cm) & 38.6 & 38.37 \\
\hline
\end{tabular}


We compared our study with other studies as depicted in Table 3 and found fewer infection and thrombotic complications in our study, which could be due to differences in the sample size, undertaking aseptic precautions while performing the procedure and regular follow up for dressing.

\begin{tabular}{|c|c|c|c|c|c|}
\hline Charactersitics & $\begin{array}{l}\text { Current } \\
\text { Study }\end{array}$ & $\begin{array}{l}\text { Sulay S } \\
\text { et al }{ }^{[15]}\end{array}$ & $\begin{array}{c}\text { Ghulam Haider } \\
\text { et al }^{[16]}\end{array}$ & $\begin{array}{l}\text { Walshe } \\
\text { et al }{ }^{[17]}\end{array}$ & $\begin{array}{l}\text { S. Yap } \\
\text { et al }^{[3]}\end{array}$ \\
\hline Study design & Prospective & Prospective & Prospective & Prospective & Retrospective \\
\hline No of patients & 461 & 79 & 146 & 351 & 88 \\
\hline Total catheter days & 57438 & 10,868 & 3329 & 10,562 & 6872 \\
\hline Meandwell time days & 124 & 129 & 23.81 & 30 & 45 \\
\hline $\begin{array}{l}\text { Infection } \\
\text { complications/ } \\
1000 \text { catheter days }\end{array}$ & 0.27 & 0.64 & 11.1 & 4.6 & 0.7 \\
\hline $\begin{array}{l}\text { Thrombotic } \\
\text { complications/ } \\
1000 \text { catheter days }\end{array}$ & 0.22 & 0.27 & 0.3 & 1.14 & 0.7 \\
\hline
\end{tabular}

The prudence of this study is that, this study is one of a kind in Indian population, where a considerable number of patients were studied for infection and thrombotic complications of PICCs, however, more studies are needed to know about the risk factors of the infection and thrombotic complications. Smoking and high BMI are significantly contributing to the high rate of the PICC line related deep vein thrombosis (DVT). There is no consensus on the role of prophylactic anticoagulation to reduce its incidence. Further study is required to generate risk prediction models to identified patients at higher risk for PICC line related DVT. ${ }^{18}$

\section{CONCLUSION}

In Indian cancer care, PICCs are becoming common for the administration of chemotherapy as well as for basic supportive care. Here, we conducted the study which showed lesser rate of infection and thrombotic complications in cancer patients however infection complication being more common in hematological cancer patients than solid cancer patients who had more thrombotic complications. However, more prospective studies are needed to identify the risk factors and prophylactic measures to prevent infection and thrombotic complications related to PICCs.

\section{LIMITATIONS OF THE STUDY}

This study is conducted only in cancer patients and not in non cancer patients. Hence we are not able to analyse and compare complications of PICCs in cancer patients with non cancer patients.

\section{ACKNOWLEDGEMENTS \\ None}

\section{CONFLICT OF INTEREST}

None

\section{REFERENCES}

1. Hoshal VL "Total intravenous nutrition with peripherally inserted silicone elastomer central venous catheters". Arch Surg $1975 ; 110$ (5): 644-6.

2. Chu FS, Cheng VC, Law MW, Tso WK. Efficacy and complications in peripherally inserted central catheter insertion: a study using 4-Fr non-valved catheters and a single infusate. Australas Radiol 2007; 51: 453-7.

3. Yap S, Karapetis C, Lerose S, Iyer S, Koczwara B. Reducing the risk of peripherally inserted central catheter line complications in oncology setting. Eur J Cancer Care (Engl) 2006; 15: 342-7.

4. Bishop L, Dougherty L, Bodenham A, Mansi J, Crowe P, Kibbler C, et al. Guidelines on the insertion and management of central venous access devices in adults. IntI J Lab Hematol 2007; 29: 261-78.

5. Chopra, V., O'Horo, J. C., Rogers, M. A., Maki, D. G. \& Safdar, N. The risk of bloodstream infection associated with peripherally inserted central catheters compared with central venous catheters in adults: a systematic review and meta-analysis. Infect Control Hosp Epidemiol 2013;34, 908-18

6. Gunst, M. et al. Peripherally inserted central catheters may lower the incidence of catheter-related blood stream infections in patients in surgical intensive care units. Surg Infect (Larchmt) 2011; 12, 279-82

7. Maki, D. G., Kluger, D. M. \& Crnich, C. J. The risk of bloodstream infection in adults with different intravascular devices: a systematic review of 200 published prospective studies. Mayo Clin Proc 2006; $81,1159-71$

8. Al Raiy, B. et al. Peripherally inserted central venous catheters in the acute care setting: A safe alternative to high-risk short-term central venous catheters. Am J Infect Control 2010;38, 149-53

9. Ajenjo MC, Morley JC, Russo AJ, McMullen KM, Robinson C, Williams $\mathrm{RC}$, et al. Peripherally inserted central venous catheter-associated bloodstream infections in hospitalized adult patients. Infect Control Hosp Epidemiol. 2011;32(2):125-30. doi: 10.1086/657942.
10. Chopra V, Ratz D, Kuhn L, Lopus T, Chenoweth C, Krein S. PICCassociated bloodstream infections: prevalence, patterns, and predictors. Am J Med. 2014;127(4):319-28. doi: 10.1016/j.amjmed. 2014.01.001.

11. Gao, Y. et al. The incidence and risk factors of peripherally inserted central catheter-related infection among cancer patients. Ther Clin Risk Manag2015;11, 863-71

12. Delphine Grau et al Complications with peripherally inserted central catheters (PICCS) used in hospitalized patients and outpatients: a prospective cohort study Antimicrobial Resistance \& Infection Control20176:18 https://doi.org/10.1186/s13756-016-0161-0

13. Herc E et al. A Model to Predict Central-Line-Associated Bloodstream Infection Among Patients With Peripherally Inserted Central Catheters: The MPC Score. Infection control hospital epidemiology , 2017 Oct;38(10):1155-1166. doi: 10.1017/ice.2017.167. Epub 2017 Aug 15

14. Gallieni M, Pittiruti M, Biffi R. Vascular access in oncology patients. CA Cancer J Clin. 2008;58(6):323-346.

15. Sulav Sapkota et al Analysis of Use and Outcomes of Peripherally Inserted Central Catheter (PICC-Line) in Hemato-Oncological Patients Journal of Cancer Therapy, 2018, 9, 35-41

16. Ghulam Haider Determination of complication rate of PICC lines in Oncological Patients .J Pak Med Assoc 2009;vol.59;no.10.

17. Walshe LJ, Malak SF, Eagon J, Sepkowitz KA. Complication rates among cancer patients with peripherally inserted central catheters. Clin Oncol 2002; 20: 3276-81.

18. Osamah Al-Asadi,Manar A, Hany E. Predictive risk factors of venous thromboembolism (VTE) associated with peripherally inserted central catheters (PICC) in ambulant solid cancer patients: retrospective single Centre cohort study. Thromb J. 2019;17: 2 . 